\title{
A NOTE ON SPECIAL VALUES OF CERTAIN DIRICHLET $L$-FUNCTIONS
}

\author{
B. RAMAKRISHNAN
}

(Received 23 June 2010)

\begin{abstract}
In Gun and Ramakrishnan ['On special values of certain Dirichlet $L$-functions', Ramanujan J. 15 (2008), 275-280], we gave expressions for the special values of certain Dirichlet $L$-function in terms of finite sums involving Jacobi symbols. In this note we extend our earlier results by giving similar expressions for two more special values of Dirichlet $L$-functions, namely $L\left(-1, \chi_{m}\right)$ and $L\left(-2, \chi_{-m^{\prime}}\right)$, where $m, m^{\prime}$ are square-free integers with $m \equiv 1 \bmod 8$ and $m^{\prime} \equiv 3 \bmod 8$ and $\chi_{D}$ is the Kronecker symbol $\left(\frac{D}{)}\right)$. As a consequence, using the identities of Cohen ['Sums involving the values at negative integers of $L$-functions of quadratic characters', Math. Ann. 217 (1975), 271-285], we also express the finite sums with Jacobi symbols in terms of sums involving divisor functions. Finally, we observe that the proof of Theorem 1.2 in Gun and Ramakrishnan (as above) is a direct consequence of Equation (24) in Gun, Manickam and Ramakrishnan ['A canonical subspace of modular forms of half-integral weight', Math. Ann. 347 (2010), 899-916].
\end{abstract}

2000 Mathematics subject classification: primary 11E25, 11M06; secondary 11F11, 11D85.

Keywords and phrases: Dirichlet $L$-functions, sums of squares.

\section{Introduction}

For a positive integer $k$, let $r_{k}(n)$ denote the number of ways a positive integer $n$ can be written as a sum of $k$ squares; and for $s \in \mathbb{C}$, and $\chi$ a Dirichlet character modulo $m \in \mathbb{N}$, let $L(s, \chi)$ denote the Dirichlet $L$-function associated with the character $\chi$. Further, let $\chi_{D}$ denote the Kronecker symbol defined by $\chi_{D}(n)=\left(\frac{D}{n}\right)$, where $D$ is the discriminant of a quadratic field. In our earlier work with Gun [6], we proved the conjectures of Cooper [3] on certain formulas for $r_{9}\left(m_{1}\right)$ and $r_{11}\left(m_{2}\right), m_{1}, m_{2}$ square-free integers with $m_{1} \equiv 5 \bmod 8, m_{2} \equiv 7 \bmod 8$, expressing them as finite sums involving Jacobi symbols. Then, using the formula for $r_{k}(n)$ obtained in [7], we derived the expressions for the special values of the $L$-functions, namely, $L\left(-3, \chi_{m_{1}}\right)$ and $L\left(-4, \chi_{-m_{2}}\right)$ in terms of finite sums involving Jacobi symbols.

In this note, we first observe that [6, proof of Theorem 1.2] simplifies (in fact becoming direct) if we apply [5, Theorem 3.2]. This was demonstrated in [5, Equation (24)], which we reproduce here. If $k \in\{2,3,4,5,7\}$ and $t$ is a square-

(C) 2010 Australian Mathematical Publishing Association Inc. 0004-9727/2010 \$16.00 
free integer with $t \equiv 1 \bmod 4,(-1)^{k} t>0$ and $\left(\frac{t}{2}\right)=-\left(\frac{2}{2 k+1}\right)$, then

$$
r_{2 k+1}(|t|)=\frac{L\left(1-k,\left(\frac{4 t}{\cdot}\right)\right)}{\zeta(1-2 k)},
$$

where $\zeta(s)$ is the Riemann zeta function. Note that

$$
L\left(1-k,\left(\frac{4 t}{\cdot}\right)\right)=\left(1-\left(\frac{t}{2}\right) 2^{k-1}\right) L\left(1-k, \chi_{t}\right)
$$

and

$$
\zeta(1-2 k)=\frac{-B_{2 k}}{2 k}
$$

where $B_{k}$ is the $k$ th Bernoulli number. Next, it is known that $r_{5}(m)$ and $r_{7}\left(m^{\prime}\right)$ for certain class of square-free integers $m, m^{\prime}$ can be expressed in terms of the finite sums involving Jacobi symbols. So, combining with the identity (1.1), we obtain similar expressions for $L\left(-1, \chi_{m}\right), m \equiv 1 \bmod 8$ and $L\left(-2, \chi_{-m^{\prime}}\right), m^{\prime} \equiv 3 \bmod 8$. From the work of Cohen [2], we know that one can express $r_{5}\left(D_{1}\right)$ and $r_{7}\left(D_{2}\right)$ in terms of $L\left(-1, \chi_{D}\right)$ and $L\left(-2, \chi_{-D_{2}}\right)$ respectively, and sums involving divisor sums, where $D_{1}$ and $-D_{2}$ are fundamental discriminants. Combining the above-mentioned identities of Cohen with our present results yields relations between the sums involving Jacobi symbols and divisor sums.

\section{New results}

We now state and prove our new results.

THEOREM 2.1. Let $m>1$ and $m^{\prime}>0$ be square-free integers such that $m \equiv 1 \bmod 8$ and $m^{\prime} \equiv 3 \bmod 8$. Then

$$
\begin{gathered}
L\left(-1, \chi_{m}\right)=\frac{2}{3} \sum_{j=1}^{(m-1) / 2}\left(\frac{j}{m}\right) j, \\
L\left(-2, \chi_{-m^{\prime}}\right)=-\frac{2}{27} \sum_{j=1}^{\left(m^{\prime}-1\right) / 2}\left(\frac{j}{m^{\prime}}\right)\left(m^{\prime 2}-6 j^{2}\right) .
\end{gathered}
$$

PROOF. It is known that for $m>1, m^{\prime}>0$ square-free with $m \equiv 1 \bmod 8$ and $m^{\prime} \equiv$ $3 \bmod 8$,

$$
\begin{gathered}
r_{5}(m)=-80 \sum_{j=1}^{(m-1) / 2}\left(\frac{j}{m}\right) j, \\
r_{7}\left(m^{\prime}\right)=\frac{280}{3} \sum_{j=1}^{\left(m^{\prime}-1\right) / 2}\left(\frac{j}{m^{\prime}}\right)\left(m^{\prime 2}-6 j^{2}\right)
\end{gathered}
$$


(see, for example, [4, pp. 12-13]). Substituting $k=2,3$ in (1.1), we get for positive square-free $m, m^{\prime}$ with $m \equiv 1 \bmod 8, m^{\prime} \equiv 3 \bmod 8$,

$$
\begin{aligned}
r_{5}(m) & =\frac{L\left(-1,\left(\frac{4 m}{\cdot}\right)\right)}{\zeta(-3)} \\
r_{7}\left(m^{\prime}\right) & =\frac{L\left(-2,\left(\frac{-4 m^{\prime}}{\cdot}\right)\right)}{\zeta(-5)} .
\end{aligned}
$$

But we have

$$
\zeta(-3)=\frac{-B_{4}}{4}=\frac{1}{120}, \quad \zeta(-5)=\frac{-B_{6}}{6}=\frac{-1}{252}
$$

and

$$
\begin{gathered}
L\left(-1,\left(\frac{4 m}{\cdot}\right)\right)=-L\left(-1, \chi_{m}\right), \\
L\left(-2,\left(\frac{-4 m^{\prime}}{\cdot}\right)\right)=5 \cdot L\left(-2, \chi_{-m^{\prime}}\right) .
\end{gathered}
$$

Hence,

$$
\begin{gathered}
r_{5}(m)=-120 L\left(-1, \chi_{m}\right), \\
r_{7}\left(m^{\prime}\right)=-1260 L\left(-2, \chi_{-m^{\prime}}\right) .
\end{gathered}
$$

Now combining (2.2) and (2.4) we get the required identities.

In [2, Corollary 3.2], Cohen showed the following identity:

$$
L\left(-1, \chi_{D}\right)=\frac{1}{120} r_{5}(D)-\frac{1}{6} \sum_{\substack{|j|<\sqrt{D} \\ D-j^{2} \text { odd }}} \sigma\left(D-j^{2}\right),
$$

where $D>0$ is the discriminant of a real quadratic field and $\sigma(n)$ is the sum of the divisors of $n$. Taking $D=m>1$ with $m$ square-free and $m \equiv 1 \bmod 8$, and using the first equations of (2.1) and (2.2), we obtain the following corollary.

COROLlary 2.2. Let $m>1$ be a square-free integer with $m \equiv 1 \bmod 8$. Then

$$
\sum_{\substack{|j|<\sqrt{m} \\ m-j^{2} \text { odd }}} \sigma\left(m-j^{2}\right)=-8 \sum_{j=1}^{(m-1) / 2}\left(\frac{j}{m}\right) j .
$$

Using [1, Theorem 3] and using the first equations of (2.1) and (2.2), we obtain another identity.

COROllary 2.3. Let $m>1$ be a square-free integer with $m \equiv 1 \bmod 8$. Then

$$
\sum_{|j|<\sqrt{m}} \sigma\left(m-j^{2}\right)=\frac{-70}{3} \sum_{j=1}^{(m-1) / 2}\left(\frac{j}{m}\right) j .
$$


In the following we obtain an expression for the convolution sum of $r_{3}(n)$ and $\sigma(n)$ in terms of the finite sum that appears in Theorem 2.1 (the sum in the second equation). We obtain this by combining [2, Corollary 3.2] and our identities. Let $-N$ be a negative fundamental discriminant. Then, using the second identity in [2, Corollary 3.2] and comparing the $N$ th Fourier coefficients on both the sides, we get

$$
L\left(-2, \chi_{-N}\right)=\frac{-1}{252} r_{7}(N)+\frac{1}{18} \psi_{4}(N) \sigma(N)+\frac{1}{18} \sum_{\substack{j=1 \\ N-j \text { odd }}}^{N-1} r_{3}(j) \sigma(N-j),
$$

where $\psi_{4}$ is the trivial Dirichlet character modulo 4 . Therefore, using the second equations of (2.1) and (2.2) in the above expression, we obtain the following.

COROLlary 2.4. Let $m^{\prime}$ be a square-free integer with $m^{\prime} \equiv 3 \bmod 8$. Then

$$
\sum_{\substack{j=0 \\ m^{\prime}-j \text { odd }}}^{m^{\prime}-1} r_{3}(j) \sigma\left(m^{\prime}-j\right)=\frac{16}{3} \sum_{j=1}^{\left(m^{\prime}-1\right) / 2}\left(\frac{j}{m^{\prime}}\right)\left(m^{\prime 2}-6 j^{2}\right),
$$

where we take $r_{3}(0)=1$.

Note that $r_{3}(n)$ can be expressed in terms of finite sums involving Jacobi symbols (see, for example, [4, Theorem 3]).

\section{References}

[1] P. T. Bateman and M. I. Knopp, 'Some new old-fashioned modular identities', Ramanujan J. 2 (1998), 247-269.

[2] H. Cohen, 'Sums involving the values at negative integers of $L$-functions of quadratic characters', Math. Ann. 217 (1975), 271-285.

[3] S. Cooper, 'Sums of five, seven and nine squares', Ramanujan J. 6 (2002), 469-490.

[4] S. Cooper and M. Hirschhorn, 'On the number of primitive representations of integers as sums of squares', Ramanujan J. 13 (2007), 7-25.

[5] S. Gun, M. Manickam and B. Ramakrishnan, 'A canonical subspace of modular forms of halfintegral weight', Math. Ann. 347 (2010), 899-916.

[6] S. Gun and B. Ramakrishnan, 'On special values of certain Dirichlet $L$-functions', Ramanujan J. 15 (2008), 275-280.

[7] S. Gun and B. Ramakrishnan, 'On the representation of integers as sums of an odd number of squares', Ramanujan J. 15 (2008), 367-376.

\section{B. RAMAKRISHNAN, Harish-Chandra Research Institute, Chhatnag Road, Jhunsi, Allahabad 211 019, India e-mail: ramki@hri.res.in}

